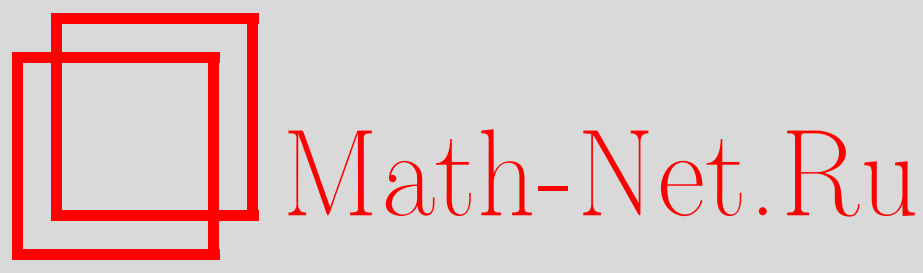

В. А. Андреев, Корреляционные неравенства Белла, TMФ, 2009, том 158, номер 2, 234-249

DOI: https://doi.org/10.4213/tmf6311

Использование Общероссийского математического портала Math-Net.Ru подразумевает, что вы прочитали и согласны с пользовательским соглашением http://www . mathnet.ru/rus/agreement

Параметры загрузки :

IP: 54.89 .56 .158

26 апреля 2023 г., 13:26:02

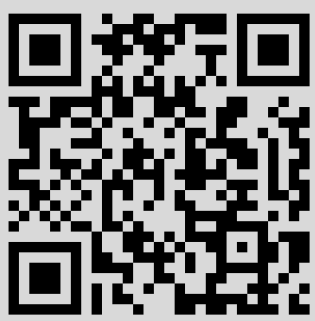




\section{КОРРЕЛЯЦИОННЫЕ НЕРАВЕНСТВА БЕЛЛА}

Рассматриваются неравенство Белла и неравенство Белла-Клаузера-ХорнеШимони-Хольта для двухчастичных спиновых состояний. Известно, что эти неравенства нарушаются при экспериментальной проверке. Показано, что это можно объяснить тем, что эти неравенства доказаны для корреляционных функций случайных величин, которые никак не связаны друг с другом, а при проверке используются корреляционные функции, в которых случайные величины относятся к паре частиц, образующих двухчастичное состояние. В случае зацепленных состояний эти случайные функции зависимы и их коэффициент корреляции не равен нулю. Приведены неравенства, в которые этот коэффициент корреляции входит явным образом. Для факторизуемых и сепарабельных состояний они совпадают с обычными неравенствами Белла и Белла-Клаузера-Хорне-Шимони-Хольта.

Ключевые слова: квантовая механика, неравенства Белла, спиновые состояния, коэффициент корреляции, скрытые параметры, нелокальность, вероятность.

\section{1. ВВЕДЕНИЕ}

Проблема существования в квантовой механике скрытых параметров имеет давнюю историю [1], [2]. Одними из главных объектов, которые используются при анализе этой проблемы, являются неравенства Белла и их обобщения [3]-[5]. Тот факт, что они нарушаются при экспериментальной проверке, обычно рассматривают как свидетельство отсутствия скрытых параметров [6], [7]. Одновременно с этим обычно обсуждают и проблему локальности в квантовой механике. В настоящей работе анализируются допущения, которые делаются при выводе этих неравенств, и те заключения, которые можно вывести из факта их нарушения.

Следует отметить, что при построении некоторых неравенств этого типа явным образом не делается никаких предположений о характере модели. Просто данные измерений упорядочиваются некоторым специальным способом. В результате такого упорядочивания получают некоторые формулы для измеряемых величин, которые и сравнивают с результатами измерений [8], [9]. В этом случае становится вообще непонятным, почему возникает несовпадение расчетов с измерениями, ведь

* Физический институт им. П. Н. Лебедева РАН, Москва, Россия. Email: andrvlad@yandex.ru 
вроде бы сравниваются одни и те же величины, только упорядоченные разным образом. Последние результаты, посвященные этой проблематике можно найти в обзорах [10], [11].

Следует учитывать, что не существует последовательной теории микромира, основанной на предположении о существовании скрытых параметров. Поэтому все рассуждения основываются на некоторых гипотезах и предположениях, которые, как правило, делаются с целью получить некоторую конкретную оценку [12].

\section{2. ВЕРОЯТНОСТНОЕ НЕРАВЕНСТВО БЕЛЛА}

Обычно рассуждения основываются на следующих предположениях. Рассматривается одночастичное спиновое состояние $\psi$. Измеряется величина проекции спина этого состояния на некоторую ось, заданную единичным вектором $\vec{a}$. Проводится серия таких измерений, и результат измерений представляется в виде функции $A(\psi, \vec{a})$, принимающей значения \pm 1 . При этом если проекция спина состояния $\psi$ направлена в положительном направлении оси $\vec{a}$, то $A(\psi, \vec{a})=1$; если проекция спина состояния $\psi$ направлена в отрицательном направлении оси $\vec{a}$, то $A(\psi, \vec{a})=-1$.

Величина $A(\psi, \vec{a})$ является дихотомной случайной функцией, ее структура определяется состоянием $\psi$ и вектором $\vec{a}$. Выбирая различные одночастичные состояния и векторы, на которые проецируются их спины, можно получить любые дихотомные случайные функции. Для них можно доказать некоторое неравенство, которое называют неравенством Белла. Мы будем называть его здесь вероятностным неравенством Белла (ВНБ). Приведем, следуя [7], его вывод.

Пусть $\wp=(\Omega, \mathcal{F}, P)$ - колмогоровское вероятностное пространство. Здесь $\Omega-$ множество элементарных событий, каждое из них характеризуется набором параметров $\lambda, \mathcal{F}-\sigma$-алгебра подмножеств множества $\Omega$, а $P-\sigma$-аддитивная мера на алгебре $\mathcal{F}$, нормированная условием $P(\Omega)=1$. Случайные величины определяются как $P$-измеримые функции $\xi:(\Omega, \mathcal{F}) \rightarrow(\mathbb{R}, \mathcal{B})$. Здесь $\mathbb{R}-$ множество вещественных чисел, а $\mathcal{B}$ - борелевская $\sigma$-алгебра множеств на действительной прямой.

Рассмотрим случай, когда все случайные величины являются дихотомными и принимают значения \pm 1 . Для любых двух таких случайных величин можно определить значение их корреляции

$$
M(A, B)=\int_{\Omega} A(\lambda) B(\lambda) d P(\lambda) .
$$

ПреДЛОЖЕНИЕ. Для любъх случайных величин $A, B, C$ корреляиии удовлетворяют неравенству

$$
|M(A, B)-M(C, B)| \leqslant 1-M(A, C) .
$$

ДоказАтельство. Рассмотрим величину $M(A, B)-M(C, B)$. Используя представление $(1)$ и соотношение $A^{2}(\lambda)=1$, ее можно записать в виде

$$
\begin{aligned}
& M(A, B)-M(C, B)=\int_{\Omega} A(\lambda) B(\lambda) d P(\lambda)-\int_{\Omega} C(\lambda) B(\lambda) d P(\lambda)= \\
& \quad=\int_{\Omega}(A(\lambda)-C(\lambda)) B(\lambda) d P(\lambda)=\int_{\Omega}(1-A(\lambda) C(\lambda)) A(\lambda) B(\lambda) d P(\lambda) .
\end{aligned}
$$


Теперь, взяв модуль величины (3), получим неравенство (2):

$$
\begin{aligned}
|M(A, B)-M(C, B)| & =\left|\int_{\Omega}(1-A(\lambda) C(\lambda)) A(\lambda) B(\lambda) d P(\lambda)\right| \leqslant \\
& \leqslant \int_{\Omega}(1-A(\lambda) C(\lambda)) d P(\lambda)=1-M(A, C) .
\end{aligned}
$$

Данные рассуждения, приводящие к неравенству (2), часто рассматривают как доказательство неравенства Белла. Но пока это чисто формальное соотношение, полученное в рамках теории вероятностей. Чтобы рассматривать его как физическое утверждение, необходимо придать входящим в него величинам физический смысл. С этой целью проанализируем процедуру, с помощью которой Белл получил свое неравенство, которое действительно похоже на неравенство (2).

Белл в работе [3] исходит из того, что имеется некоторое одночастичное спиновое состояние $\psi$, которое помимо обычных спиновых переменных характеризуется также некоторым набором параметров, обозначающихся буквой $\lambda$. Предполагается, что, зная конкретные значения этих скрытых параметров, можно однозначно предсказать величину проекции спина состояния $\psi$ на любую ось. Значения параметров $\lambda$ заполняют некоторую область в пространстве, размерность которого не уточняется. Каждая конкретная система характеризуется распределением $\rho(\lambda)$, которое является плотностью вероятности обнаружить у этой системы набор параметров $\lambda$. Выполняется соотношение

$$
\int \rho(\lambda) d \lambda=1 .
$$

Введем функцию $A(\vec{a}, \lambda)$. Если проекция спина состояния $\psi$ направлена в положительном направлении оси $\vec{a}$, то $A(\vec{a}, \lambda)=1$; если проекция спина состояния $\psi$ направлена в отрицательном направлении оси $\vec{a}$, то $A(\vec{a}, \lambda)=-1$. Эта функция не является случайной величиной. Выбор оси $\vec{a}$ и набора параметров $\lambda$ однозначно определяет ее значение. Случайным при таком подходе является выбор значения параметров $\lambda$, а вероятность того или иного выбора задается распределением $\rho(\lambda)$.

Если имеются две частицы, то одной из них сопоставляется функция $A(\vec{a}, \lambda)$, а второй - функция $B(\vec{b}, \lambda)$. Величина их спиновой корреляции определяется соотношением

$$
E(\vec{a}, \vec{b})=\int \rho(\lambda) A(\vec{a}, \lambda) B(\vec{b}, \lambda) d \lambda .
$$

При этом делается предположение, что функции $A(\vec{a}, \lambda)$ и $B(\vec{b}, \lambda)$ независимы, т.е. значение функции $A(\vec{a}, \lambda)$ не зависит от того, какое значение принимает функция $B(\vec{b}, \lambda)$ и наоборот. Такое утверждение в явном виде содержится в работе [5].

В работе [3] рассматривается величина $E(\vec{a}, \vec{b})-E(\vec{a}, \vec{c})$. Используя представление (6), ее можно записать в виде

$$
\begin{aligned}
E(\vec{a}, \vec{b})-E(\vec{a}, \vec{c}) & =\int \rho(\lambda) A(\vec{a}, \lambda) B(\vec{b}, \lambda) d \lambda-\int \rho(\lambda) A(\vec{a}, \lambda) B(\vec{c}, \lambda) d \lambda= \\
& =\int \rho(\lambda)[A(\vec{a}, \lambda) B(\vec{b}, \lambda)-A(\vec{a}, \lambda) B(\vec{c}, \lambda)] d \lambda= \\
& =\int \rho(\lambda) A(\vec{a}, \lambda) B(\vec{b}, \lambda)[1-B(\vec{b}, \lambda) B(\vec{c}, \lambda)] d \lambda .
\end{aligned}
$$


Взяв модуль от обеих частей соотношения (7), получим неравенство

$$
|E(\vec{a}, \vec{b})-E(\vec{a}, \vec{c})| \leqslant 1-\int \rho(\lambda) B(\vec{b}, \lambda) B(\vec{c}, \lambda) d \lambda=1-E(\vec{b}, \vec{c}) .
$$

Неравенство Белла (8) отличается от ВНБ (2) тем, что в нем случайные величины имеют конкретный физический смысл, а именно представляют собой проекции спинов одночастичных состояний на ту или иную ось, соответствующие всевозможным значениям скрытых параметров. Можно считать, что эти величины возникают следующим образом. Имеется источник одночастичных спиновых состояний. Измеряя проекции спинов этих частиц на некоторую ось $\vec{a}$, получаем величину $A(\vec{a})$. Если предположить, что существуют скрытые параметры, которые в каждом случае однозначно определяют значения этих проекций, то величина $A(\vec{a}, \lambda)$ является детерминированной функцией своих переменных, но, поскольку нам они не известны, для нас она выглядит как случайная функция. Таким способом, измеряя проекции спинов одночастичных состояний, можно получить набор случайных величин

$$
N F=\{A(\vec{a}, \lambda), B(\vec{b}, \lambda), C(\vec{c}, \lambda), \ldots\}
$$

Никакой связи между этими величинами, вообще говоря, нет. Даже если источник испускает двухчастичные коррелированные состояния, то проекции спинов каждого из этих состояний измеряются независимо, и в наборе (9) не отмечено, какие из входящих в него величин относятся к одним и тем же парам коррелированных состояний. Важным является также то, что неравенства (2) и (8) доказаны для трех произвольных случайных величин $A, B, C$ из набора (9).

Общеизвестно утверждение, что эти неравенства нарушаются при экспериментальной проверке [13]-[18]. Предлагались различные объяснения данного факта. В книге [7] дано вероятностное объяснение нарушения неравенств Белла. В ней совершенно правильно указано, что две случайные величины $A(\vec{a}, \lambda)$ и $A^{\prime}(\vec{a}, \lambda)$, полученные с помощью двух разных процедур измерения, - это две разные случайные величины, и их нельзя отождествлять друг с другом при подстановке в неравенства (2), (8). При экспериментальной проверке каждая из случайных величин, входящих в эти неравенства, измеряется самостоятельно, т.е. наряду с величинами $A(\vec{a}, \lambda), B(\vec{b}, \lambda), C(\vec{c}, \lambda)$ в них входят величины $A^{\prime}(\vec{a}, \lambda), B^{\prime}(\vec{b}, \lambda), C^{\prime}(\vec{c}, \lambda)$, и все эти шесть случайных величин, вообще говоря, различны. Таким образом, неравенства (2), (8) доказаны для трех случайных величин, а при их проверке используется шесть, отсюда и возникает несовпадение теории с экспериментом. В книге [7] были введены коэффициенты, численно характеризующие величину различия таких статистических ансамблей, и построено обобщение неравенства Белла, в которое эти коэффициенты входят явным образом. Мы согласны с тем, что это одна из причин нарушения неравенств Белла, но есть и другие.

В настоящей работе мы развиваем другой подход к проблеме нарушения неравенств Белла. Главной причиной такого нарушения мы считаем то, что неравенства (2), (8) доказаны для корреляционных функций (6) двух произвольных случайных величин, а при экспериментальной проверке используются корреляционные функции специального вида, у которых под интегралом в выражении (6) стоят случайные величины, относящиеся к коррелированной паре одночастичных состояний. 
Для таких корреляционных функций неравенства (2), (8), вообще говоря, не выполняются. Связь между случайными величинами, относящимися к одной и той же коррелированной паре, задается коэффициентами корреляции. В нашей работе построены неравенства, включающие эти коэффициенты корреляции. Мы называем их корреляционными неравенствами Белла. Они не нарушаются ни для каких двухчастичных состояний и в случае, когда коэффициенты корреляции равны нулю, переходят в обычные неравенства Белла.

Кроме того, в работе развита техника редукции матрицы плотности, с помощью которой получено явное выражение для коэффициента корреляции через элементы матрицы плотности.

\section{3. СПИНОВЫЕ КОРРЕЛЯЦИИ}

Вернемся к неравенству Белла (8). Как уже говорилось, при его доказательстве предполагается, что в него входят три произвольные случайные величины $A, B, C$. Их корреляция определяется соотношением (6).

Посмотрим теперь, как осуществляется проверка этого неравенства. При экспериментальной проверке используется источник, испускающий двухчастичные спиновые состояния. Предполагается, что все эти состояния идентичны. В этом случае $E(\vec{a}, \vec{b})$ - это величина спиновой корреляции для частиц, образующих такую пару. Определяется она следующим образом. В пространстве выбираются два направления, задаваемые векторами $\vec{a}, \vec{b}$, и измеряются значения проекций спинов частиц на эти направления: первой частицы - на ось $\vec{a}$, а второй частицы - на ось $\vec{b}$. Проводится серия из $N$ измерений с такими парами коррелированных частиц. Результаты этих измерений для $i$-й пары обозначим $v_{i}(\vec{a}), w_{i}(\vec{b})$. Величины $v_{i}(\vec{a}), w_{i}(\vec{b})$ равны \pm 1 , знак зависит от того, куда направлен спин.

Среднее значение удвоенных проекций спина первой частицы на ось $\vec{a}$, полученное в результате $N$ измерений, определяется величиной

$$
E(\vec{a})=2 S^{(1)}(\vec{a})=\frac{1}{N} \sum_{i=1}^{N} v_{i}(\vec{a}),
$$

а среднее значение удвоенных проекций спина второй частицы на ось $\vec{b}$, полученное в результате $N$ измерений, определяется величиной

$$
E(\vec{b})=2 S^{(2)}(\vec{b})=\frac{1}{N} \sum_{j=1}^{N} w_{j}(\vec{b}) .
$$

Среднее значение произведений $v_{i}(\vec{a}) w_{i}(\vec{b})$, полученное в результате $N$ измерений, определяет величину спиновой корреляции:

$$
E(\vec{a}, \vec{b})=\frac{1}{N} \sum_{i=1}^{N} v_{i}(\vec{a}) w_{i}(\vec{b}) .
$$

В этом определении важно то, что в данной сумме стоят произведения величин $v_{i}(\vec{a}), w_{i}(\vec{b})$, полученных при измерении проекций спинов частиц, относящихся $\mathrm{K}$ 
одной коррелированной паре, т.е. это те две частицы, которые изначально образовывали двухчастичное состояние, испущенное источником. Величины $v_{i}(\vec{a}), w_{i}(\vec{b})$ измеряются практически одновременно. Мы будем называть последовательности данных $V(\vec{a})=\left\{v_{i}(\vec{a}) ; i=1, \ldots, N\right\}, W(\vec{b})=\left\{w_{j}(\vec{b}) ; j=1, \ldots, N\right\}$, полученных таким образом, данными, полученными в одной серии измерений. Если же величины $v_{i}(\vec{a}), w_{i}(\vec{b})$ получены при измерении проекций спинов частиц, входящих в разные пары коррелированных частиц, т.е. они измерены в разное время, то последовательности данных $V(\vec{a})=\left\{v_{i}(\vec{a}) ; i=1, \ldots, N\right\}, W(\vec{b})=\left\{w_{j}(\vec{b}) ; j=1, \ldots, N\right\}$, построенные таким образом, мы будем называть последовательностями, полученными в разных сериях измерений.

В неравенство Белла (8) входят три корреляционных средних. Поэтому при его экспериментальной проверке проводится три серии измерений, которые дают значения шести случайных величин

$$
\begin{array}{ll}
V^{1}(\vec{a})=\left\{v_{i}^{1}(\vec{a}) ; i=1, \ldots, N\right\}, & W^{1}(\vec{b})=\left\{w_{i}^{1}(\vec{b}) ; i=1, \ldots, N\right\}, \\
V^{2}(\vec{a})=\left\{v_{i}^{2}(\vec{c}) ; i=1, \ldots, N\right\}, & W^{2}(\vec{b})=\left\{w_{i}^{2}(\vec{b}) ; i=1, \ldots, N\right\}, \\
V^{3}(\vec{a})=\left\{v_{i}^{3}(\vec{a}) ; i=1, \ldots, N\right\}, & W^{3}(\vec{b})=\left\{w_{i}^{3}(\vec{c}) ; i=1, \ldots, N\right\},
\end{array}
$$

где верхние индексы $1,2,3$ обозначают номер серии измерений.

Обсудим некоторые свойства этих случайных величин.

Во-первых, поскольку предполагается, что пары частиц, которые испускает источник, идентичны, величина (10), определяющая среднее значение удвоенной проекции спина первой частицы на ось $\vec{a}$, принимает одно и то же значение при всех сериях измерений. Поэтому наборы $\left\{v_{i}^{1}(\vec{a}) ; i=1, \ldots, N\right\}$ и $\left\{v_{i}^{2}(\vec{a}) ; i=1, \ldots, N\right\}$ содержат одинаковое количество +1 и -1 , но расположены они в них в разном порядке. Поэтому случайные величины $V^{1}(\vec{a})$ и $V^{2}(\vec{a})$, значения которых задаются этими наборами, вообще говоря различны, но между ними можно установить соответствие, изменив нумерацию их значений.

Во-вторых, легко видеть, что результаты измерений проекций спинов частиц, образующих коррелированную пару, вообще говоря, не являются независимыми. Связь между ними определяется тем, что величина спиновой корреляции (12) не зависит от процедуры измерений. Поэтому, как количество +1 и -1 в наборах $\left\{v_{i}^{1}(\vec{a}) ; i=1, \ldots, N\right\},\left\{w_{i}^{1}(\vec{b}) ; i=1, \ldots, N\right\}$, так и их расположение не являются произвольными: связь между случайными величинами $V^{1}(\vec{a}), W^{1}(\vec{b})$ определяется видом самого двухчастичного состояния, а также выбором осей $\vec{a}, \vec{b}$, на которые проецируются спины образующих его частиц. В некоторых случаях эта связь может быть абсолютно жесткой, т.е. значение величины $v_{i}(\vec{a})$ однозначно определяет значение величины $w_{i}(\vec{b})$, в других случаях она может отсутствовать совсем, и тогда значение величины $v_{i}(\vec{a})$ не зависит от значения величины $w_{i}(\vec{b})$.

Ниже мы рассмотрим конкретный пример, пока же отметим следующее. Если имеются две случайные величины $V^{1}(\vec{a})$ и $W^{2}(\vec{b})$, полученные в разных сериях измерений, то соответствующее им корреляционное среднее

$$
\widetilde{E}(\vec{a}, \vec{b})=\frac{1}{N} \sum_{i=1}^{N} v_{i}^{1}(\vec{a}) w_{i}^{2}(\vec{b})
$$


вовсе не будет являться средним значением спиновой корреляции двухчастичного спинового состояния. Еще раз подчеркнем, что из всех корреляционных средних (12) только те допускают интерпретацию в качестве средних значений спиновой корреляции, у которых величины $\left\{v_{i}(\vec{a}) ; i=1, \ldots, N\right\}$ и $\left\{w_{j}(\vec{b}) ; j=1, \ldots, N\right\}$, получены в одной серии измерений. Именно их и измеряют в эксперименте. Несоблюдение данного условия и является причиной нарушений неравенства Белла.

Чтобы убедится в этом, проанализируем доказательство неравенства (8), используя обозначения (12). В этом случае получаем

$$
E(\vec{a}, \vec{b})-E(\vec{a}, \vec{c})=\frac{1}{N} \sum_{i=1}^{N} v_{i}^{1}(\vec{a}) w_{i}^{1}(\vec{b})-\frac{1}{N} \sum_{j=1}^{N} v_{j}^{2}(\vec{a}) w_{j}^{2}(\vec{c}) .
$$

Следуя той схеме доказательства, которая использовалась при доказательстве неравенства (8), теперь надо из обоих членов правой части неравенства (15) вынести за скобку числа $v_{i}^{1}(\vec{a})$ и $v_{j}^{2}(\vec{a})$. В книге [7] утверждается, что это сделать невозможно, поскольку они являются значениями двух разных случайных величин, и что именно это и приводит к нарушению неравенств Белла. Тот факт, что нарушение неравенства Белла связано с тем, что случайные величины $V^{1}(\vec{a})$ и $V^{2}(\vec{a})$, входящие в его правую часть, являются разными случайными величинами, отмечался также в работах [19]. По нашему мнению, причина нарушения неравенств Белла состоит в другом.

Действительно, $V^{1}(\vec{a})$ и $V^{2}(\vec{a})$ - две разные случайные величины, но тем не менее операцию выноса за скобку можно осуществить, поскольку наборы чисел $\left\{v_{i}^{1}(\vec{a}) ; i=1, \ldots, N\right\}$ и $\left\{v_{j}^{2}(\vec{a}) ; i=1, \ldots, N\right\}$ содержат одинаковое количество +1 и -1 , пусть они и расположены в разном порядке. Поэтому, меняя нумерацию в наборе $\left\{v_{j}^{2}(\vec{a}), i=1, \ldots, N\right\}$ и, соответственно, порядок суммирования во втором члене правой части соотношения (15), получим выражение

$$
\begin{aligned}
E(\vec{a}, \vec{b})-E(\vec{a}, \vec{c}) & =\frac{1}{N} \sum_{i=1}^{N} v_{i}^{1}(\vec{a})\left(w_{i}^{1}(\vec{b})-w_{i}^{2}(\vec{c})\right)= \\
& =\frac{1}{N} \sum_{i=1}^{N} v_{i}^{1}(\vec{a}) w_{i}^{1}(\vec{b})\left(1-w_{i}^{1}(\vec{b}) w_{i}^{2}(\vec{c})\right) .
\end{aligned}
$$

Взяв модуль обеих частей равенства (16), получим неравенство

$$
|E(\vec{a}, \vec{b})-E(\vec{a}, \vec{c})| \leqslant 1-\frac{1}{N} \sum_{i=1}^{N} w_{i}^{1}(\vec{b}) w_{i}^{2}(\vec{c}) .
$$

Второй член в правой части этого неравенства интерпретируют как величину спиновой корреляции $E(\vec{b}, \vec{c})$ и получают неравенство Белла (8). Однако такая интерпретация недопустима. Как уже было показано, только те корреляционные средние (12) допускают интерпретацию в качестве средних значений спиновой корреляции, которые построены с помощью величин, полученных в одной серии измерений. Именно несоблюдение данного условия и является истиной причиной нарушений неравенства Белла. В левой части доказанного неравенства (17) стоят средние значения спиновой корреляции, а в его правой части стоит величина, которая таковой не 
является. Но при экспериментальной проверке неравенства в обе его части подставляют настоящие измеренные значения спиновой корреляции, что и приводит к нарушению.

Строго говоря, не существует неравенства (8), у которого все три входящие в него средние величины были бы значениями спиновых корреляций. Поэтому говорить о проверке неравенства (8) смысла не имеет, проверять можно только непосредственно неравенство (17). Однако вместо неравенства (8) можно предложить другое неравенство, которое выражается через средние значения спиновых корреляций двухчастичных состояний.

\section{4. КОРРЕЛЯЦИОННЫЕ НЕРАВЕНСТВА БЕЛЛА}

Как уже отмечалось, случайные величины $V(\vec{a})$ и $W(\vec{b})$, которые описывают результаты измерений проекций спинов частиц, образующих коррелированную пару, вообще говоря, не являются независимыми. Их зависимость характеризуется коэффициентом корреляции (здесь $\rho$ - матрица плотности соответствующего двухчастичного состояния)

$$
\begin{aligned}
K_{\rho}(\vec{a}, \vec{b}) & =\frac{1 / N \sum_{i=1}^{N} v_{i}(\vec{a}) w_{i}(\vec{b})-\left(1 / N \sum_{i=1}^{N} v_{i}(\vec{a})\right)\left(1 / N \sum_{j=1}^{N} w_{j}(\vec{b})\right)}{\sqrt{1-\left(1 / N \sum_{i=1}^{N} v_{i}(\vec{a})\right)^{2}} \sqrt{1-\left(1 / N \sum_{j=1}^{N} w_{j}(\vec{b})\right)^{2}}}= \\
& =\frac{1 / N \sum_{i=1}^{N} v_{i}(\vec{a}) w_{i}(\vec{b})-E(\vec{a}) E(\vec{b})}{\sqrt{1-E^{2}(\vec{a})} \sqrt{1-E^{2}(\vec{b})}} .
\end{aligned}
$$

Если последовательности данных $\left\{v_{i}(\vec{a}) ; i=1, \ldots, N\right\},\left\{w_{j}(\vec{b}) ; j=1, \ldots, N\right\}$ получены в одной серии измерений, то коэффициент корреляции (18) принимает вид

$$
K_{\rho}(\vec{a}, \vec{b})=\frac{E(\vec{a}, \vec{b})-E(\vec{a}) E(\vec{b})}{\sqrt{1-E^{2}(\vec{a})} \sqrt{1-E^{2}(\vec{b})}} .
$$

Теперь, используя выражение (18) для коэффициента корреляции, неравенство (17) можно записать в виде

$$
|E(\vec{a}, \vec{b})-E(\vec{a}, \vec{c})| \leqslant 1-E(\vec{b}) E(\vec{c})+K_{\rho}(\vec{a}, \vec{b})\left(\sqrt{1-E^{2}(\vec{a})} \sqrt{1-E^{2}(\vec{b})}\right) .
$$

Если коэффициент корреляции равен нулю, т.е. случайные величины $V(\vec{a}), W(\vec{b})$ независимы, то из соотношения $K(\vec{a}, \vec{b})=0$ следует равенство

$$
E(\vec{a}, \vec{b})=E(\vec{a}) E(\vec{b}) .
$$

При этом корреляционное неравенство (20) переходит в неравенство Белла (8), и это тот самый случай, когда значения $v_{i}(\vec{a})$ случайной величины $V(\vec{a})$ не зависят от значений $w_{i}(\vec{b})$ случайной величины $W(\vec{b})$. В этом случае при построении спиновой корреляции (12) можно использовать последовательности данных $\left\{v_{i}(\vec{a}) ; i=1, \ldots, N\right\}$, $\left\{w_{j}(\vec{b}) ; j=1, \ldots, N\right\}$, полученные в разных сериях измерений, причем эти разные серии измерений могут относится к разным парам осей, на которые проецируются 
спины частиц. Например, вместо последовательности $\left\{w_{j}(\vec{b}) ; j=1, \ldots, N\right\}$, полученной при измерении проекций спинов на пару осей $\vec{a}, \vec{b}$, можно использовать последовательность $\left\{w_{j}(\vec{b}) ; j=1, \ldots, N\right\}$, полученную при измерении проекций спинов на пару осей $\vec{d}, \vec{b}$.

Но если коэффициент корреляции $K_{\rho}(\vec{a}, \vec{b})$ не равен нулю и случайные величины $V(\vec{a}), W(\vec{b})$ зависимы, то при построении спиновой корреляции (12) можно использовать только те последовательности данных $\left\{v_{i}(\vec{a}) ; i=1, \ldots, N\right\},\left\{w_{j}(\vec{b}) ; j=\right.$ $1, \ldots, N\}$, которые получены в одной серии измерений. Если же мы подставим в выражение (12) последовательности данных $\left\{v_{i}(\vec{a}) ; i=1, \ldots, N\right\},\left\{w_{j}(\vec{b}) ; j=\right.$ $1, \ldots, N\}$, полученные в разных сериях измерений, то возникающую сумму нельзя интерпретировать как спиновую корреляцию $E(\vec{a}, \vec{b})$ и, вообще говоря, она не будет иметь физического смысла.

Неравенство (20) должно выполняться для произвольных двухчастичных спиновых состояний. Неравенство Белла (8) является его частным случаем, оно выполняется для факторизуемых и сепарабельных состояний. Тот факт, что оно нарушается при экспериментальной проверке, означает, что в эксперименте использовались двухчастичные состояния с ненулевым коэффициентом корреляции, т.е. такие состояния, для которых выражение $\int \rho(\lambda) B(\vec{b}, \lambda) B(\vec{c}, \lambda) d \lambda$, входящее в $(8)$, нельзя интерпретировать как величину спиновой корреляции $E(\vec{b}, \vec{c})$.

Рассмотрим неравенство Белла-Клаузера-Хорне-Шимони-Хольта (БКХШХ) [5]:

$$
|E(\vec{a}, \vec{b})+E(\vec{a}, \vec{c})+E(\vec{d}, \vec{b})-E(\vec{d}, \vec{c})| \leqslant 2 .
$$

Оно выводится следующим образом. Запишем левую часть неравенства (22), используя выражение (12) для величины спиновой корреляции. В этом случае каждая из корреляций определяется с помощью суммы (12):

$$
\begin{aligned}
E(\vec{a}, \vec{b})+ & E(\vec{a}, \vec{c})+E(\vec{d}, \vec{b})-E(\vec{d}, \vec{c})= \\
=\frac{1}{N} & \left(\sum_{i=1}^{N} v_{i}^{1}(\vec{a}) w_{i}^{1}(\vec{b})+\sum_{j=1}^{N} v_{j}^{2}(\vec{a}) w_{j}^{2}(\vec{c})+\right. \\
& \left.+\sum_{k=1}^{N} v_{k}^{3}(\vec{d}) w_{k}^{3}(\vec{b})-\sum_{l=1}^{N} v_{l}^{4}(\vec{d}) w_{l}^{4}(\vec{c})\right) .
\end{aligned}
$$

Наше рассуждение отличается от обычных доказательств неравенства БКХШХ, приведенных, например, в [5], [8], [20], тем, что величины $v_{i}, w_{j}$ в правой части равенства (23) снабжены верхними индексами 1, 2, 3, 4. Они нумеруют измерения, с помощью которых были найдены значения величин спиновой корреляции, стоящие в левой части равенства (23). Эти индексы введены для того, чтобы подчеркнуть тот факт, что наборы чисел $\left\{v_{i}^{1}(\vec{a}) ; i=1, \ldots, N\right\}$ и $\left\{v_{j}^{2}(\vec{a}) ; i=1, \ldots, N\right\}$ не тождественны, они содержат одинаковое количество +1 и -1 , но эти числа расположены в них в разном порядке. Меняя нумерацию в наборах $\left\{v_{j}^{2}(\vec{a}), v_{l}^{4}(\vec{d}) ; j, l=1, \ldots, N\right\}$ и, соответственно, порядок суммирования во втором и четвертом членах правой 
части соотношения (23), запишем равенство

$$
\begin{aligned}
& E(\vec{a}, \vec{b})+E(\vec{a}, \vec{c})+E(\vec{d}, \vec{b})-E(\vec{d}, \vec{c})= \\
& =\frac{1}{N}\left(\sum_{i=1}^{N} v_{i}^{1}(\vec{a})\left(w_{i}^{1}(\vec{b})+w_{i}^{2}(\vec{c})\right)+\sum_{k=1}^{N} v_{k}^{3}(\vec{d})\left(w_{k}^{3}(\vec{b})-w_{k}^{4}(\vec{c})\right)\right) .
\end{aligned}
$$

Теперь, взяв модуль равенства (24), получим неравенство

$$
|E(\vec{a}, \vec{b})+E(\vec{a}, \vec{c})+E(\vec{d}, \vec{b})-E(\vec{d}, \vec{c})| \leqslant \frac{1}{N}\left(\sum_{i=1}^{N}\left|w_{i}^{1}(\vec{b})+w_{i}^{2}(\vec{c})\right|+\left|w_{i}^{3}(\vec{b})-w_{i}^{4}(\vec{c})\right|\right) .
$$

Стандартное рассуждение, приводящее к неравенству БКХШХ (22), состоит в следующем: $w_{i}= \pm 1$, поэтому либо $\left|w_{i}^{1}(\vec{b})+w_{i}^{2}(\vec{c})\right|=2$, a $\left|w_{i}^{3}(\vec{b})-w_{i}^{4}(\vec{c})\right|=0$, либо $\left|w_{i}^{1}(\vec{b})+w_{i}^{2}(\vec{c})\right|=0$, a $\left|w_{i}^{3}(\vec{b})-w_{i}^{4}(\vec{c})\right|=2$. При этом исходят из предположения, что при всех $i=1, \ldots, N$ имеют место равенства $w_{i}^{1}(\vec{b})=w_{i}^{3}(\vec{b}), w_{i}^{2}(\vec{c})=w_{i}^{4}(\vec{c})$. Это представляется очевидным при отсутствии у величин $w_{i}$ верхних индексов, однако на самом деле это предположение неверно. Действительно, можно так перенумеровать величины $w_{i}^{3}(\vec{b})$, что будут выполняться равенства $w_{i}^{1}(\vec{b})=w_{i}^{3}(\vec{b}), i=1, \ldots, N$, но при этом невозможно добиться, чтобы одновременно с этим выполнялись равенства $w_{i}^{2}(\vec{c})=w_{i}^{4}(\vec{c}), i=1, \ldots, N$. Дело в том, что выбор величин $w_{i}^{2}(\vec{c}), w_{i}^{4}(\vec{c})$, которые образуют пары с величинами $w_{i}^{1}(\vec{b}), w_{i}^{3}(\vec{b})$ не произволен. Величина $w_{i}^{2}(\vec{c})$ выбрана так, чтобы выполнялось условие $w_{i}^{1}(\vec{a})=w_{i}^{2}(\vec{a})$, а величина $w_{i}^{4}(\vec{c})$ выбрана так, чтобы выполнялось условие $w_{i}^{3}(\vec{d})=w_{i}^{4}(\vec{d})$, и в правой части неравенства $(25)$ стоят пары $\left|w_{i}^{1}(\vec{b})+w_{i}^{2}(\vec{c})\right|,\left|w_{i}^{3}(\vec{b})-w_{i}^{4}(\vec{c})\right|$, которые возникли именно таким образом. Величина $w_{i}^{2}(\vec{c})$ в них никак не связана с величиной $w_{i}^{4}(\vec{c})$, и если одна из этих пар равна 2, то вторая вовсе не обязана равняться нулю. Поэтому из неравенства (25) не следует неравенство (22). Неравенство (22) получается только в том случае, когда наборы чисел $\left\{v_{k}^{3}(\vec{d}) ; k=1, \ldots, N\right\}$ и $\left\{w_{k}^{4}(\vec{c}) ; k=1, \ldots, N\right\}$ образуют независимые случайные величины. Если же случайные величины зависимы и коэффициент их корреляции не равен нулю, то из соотношения (23) следует неравенство

$$
\begin{aligned}
& |E(\vec{a}, \vec{b})+E(\vec{a}, \vec{c})+E(\vec{d}, \vec{b})-E(\vec{d}, \vec{c})| \leqslant 2+K_{\rho}(\vec{a}, \vec{b})\left(\sqrt{1-E^{2}(\vec{a})} \sqrt{1-E^{2}(\vec{b})}\right)+ \\
& \quad+K_{\rho}(\vec{a}, \vec{c})\left(\sqrt{1-E^{2}(\vec{a})} \sqrt{1-E^{2}(\vec{c})}\right)+K_{\rho}(\vec{d}, \vec{b})\left(\sqrt{1-E^{2}(\vec{d})} \sqrt{1-E^{2}(\vec{b})}\right)- \\
& \quad-K_{\rho}(\vec{d}, \vec{c})\left(\sqrt{1-E^{2}(\vec{d})} \sqrt{1-E^{2}(\vec{c})}\right) .
\end{aligned}
$$

Как и в случае неравенства (20), неравенство (26) должно выполняться для произвольных двухчастичных спиновых состояний. Неравенство БКХШХ (22) является частным случаем неравенства (26), оно справедливо для факторизуемых и сепарабельных состояний. Тот факт, что оно нарушается при экспериментальной проверке, означает, что в эксперименте использовались двухчастичные состояния с ненулевым коэффициентом корреляции.

Мы будем называть неравенства (20), (26) корреляционными неравенствами Белла. В следующем разделе мы покажем, как можно выразить коэффициент корреляции (18) через элементы матрицы плотности двухчастичного состояния. 


\section{5. РЕДУКЦИЯ МАТРИЦЫ ПЛОТНОСТИ}

Пусть задано некоторое двухчастичное спиновое состояние, которое описывается матрицей плотности

$$
\rho=\left\|\rho_{i j}\right\|, \quad i, j=1,2,3,4,
$$

и образовано двумя одночастичными спиновыми состояниями. Можно измерять значения проекции спина этих состояний на некоторую ось. Предположим для простоты, что эти оси совпадают, и что это ось $Z$. В этом случае диагональные элементы матрицы плотности (27) имеют следующий смысл:

$\rho_{11}-$ вероятность того, что удвоенные проекции спинов первой и второй частиц на ось $Z$ равны +1 ;

$\rho_{22}$ - вероятность того, что удвоенная проекция спина первой частицы на ось $Z$ равна +1 , а удвоенная проекция спина второй частицы на ось $Z$ равна -1 ;

$\rho_{33}-$ вероятность того, что удвоенная проекция спина первой частицы на ось $Z$ равна -1 , а удвоенная проекция спина второй частицы на ось $Z$ равна +1 ;

$\rho_{44}-$ вероятность того, что удвоенные проекции спинов первой и второй частиц на ось $Z$ равны -1 .

Bсе эти элементы являются наблюдаемыми величинами. Для того чтобы найти их численное значение, необходимо просто измерить вероятности соответствующих проекций спинов на ось $Z$. Другими измеряемыми величинами являются значения спиновой корреляции $E(\vec{a}, \vec{b})$, которая измеряется в соответствии с формулой $(12)$. Среднее значение удвоенных проекций спина первой частицы на ось $\vec{a}$, полученное в результате $N$ измерений, определяется величиной (10), а среднее значение удвоенных проекций спина второй частицы на ось $\vec{b}$, полученное в результате $N$ измерений, определяется величиной (11).

Для величины спиновой корреляции (12) существует операторное выражение. Чтобы записать его, введем следующие обозначения. Пусть имеется одно спиновое состояние такое, что направление спина вверх по оси $Z$ обозначается как $|+\rangle$, а вниз по оси $Z-$ как $|-\rangle$. На этих векторах спиновые операторы задаются соотношениями

$$
\begin{aligned}
& S_{x}|+\rangle=\frac{1}{2}|-\rangle, \quad S_{y}|+\rangle=\frac{i}{2}|-\rangle, \quad S_{z}|+\rangle=\frac{1}{2}|+\rangle, \\
& S_{x}|-\rangle=\frac{1}{2}|+\rangle, \quad S_{y}|-\rangle=-\frac{i}{2}|+\rangle, \quad S_{z}|-\rangle=-\frac{1}{2}|-\rangle .
\end{aligned}
$$

Оператор $\hat{a}$ удвоенной проекции спина на направление, задаваемое вектором $\vec{a}=$ $\left(a_{x}, a_{y}, a_{z}\right)$, имеет вид

$$
\hat{a}=2(\vec{a}, \vec{S})=2\left(a_{x} S_{x}+a_{y} S_{y}+a_{z} S_{z}\right) .
$$

Для двухчастичных состояний можно определить оператор

$$
\hat{a} \otimes \hat{b}=4\left(\vec{a}, \vec{S}^{(1)}\right) \otimes\left(\vec{b}, \vec{S}^{(2)}\right),
$$

действующий в четырехмерном линейном пространстве, натянутом на базисные векторы

$$
\begin{array}{ll}
\Psi_{++}=|(1)+\rangle|(2)+\rangle, & \Psi_{+-}=|(1)+\rangle|(2)-\rangle, \\
\Psi_{-+}=|(1)-\rangle|(2)+\rangle, & \Psi_{--}=|(1)+\rangle|(2)+\rangle .
\end{array}
$$


При этом операторы $\vec{S}^{(1)}$ действуют на векторы $|(1)+\rangle,|(1)-\rangle$, а операторы $\vec{S}^{(2)}-$ на векторы $|(2)+\rangle,|(2)-\rangle$. Матричные элементы $\langle\psi|\hat{a} \otimes \hat{b}| \psi\rangle$ оператора (30) являются учетверенными средними значениями произведений проекций спина первой частицы на ось $\vec{a}$ и проекций спина второй частицы на ось $\vec{b}$. Оператор (30) соответствует наблюдаемой величине, называемой спиновой корреляцией. В базисе (31) оператор (30) имеет вид матрицы

$$
\left(\begin{array}{cccc}
a_{z} b_{z} & a_{z}\left(b_{x}-i b_{y}\right) & \left(a_{x}-i a_{y}\right) b_{z} & \left(a_{x}-i a_{y}\right)\left(b_{x}-i b_{y}\right) \\
a_{z}\left(b_{x}+i b_{y}\right) & -a_{z} b_{z} & \left(a_{x}-i a_{y}\right)\left(b_{x}+i b_{y}\right) & -\left(a_{x}-i a_{y}\right) b_{z} \\
\left(a_{x}+i a_{y}\right) b_{z} & \left(a_{x}+i a_{y}\right)\left(b_{x}-i b_{y}\right) & -a_{z} b_{z} & -a_{z}\left(b_{x}-i b_{y}\right) \\
\left(a_{x}+i a_{y}\right)\left(b_{x}+i b_{y}\right) & -\left(a_{x}+i a_{y}\right) b_{z} & -a_{z}\left(b_{x}+i b_{y}\right) & a_{z} b_{z}
\end{array}\right) .
$$

Наряду с оператором спиновой корреляции (30) рассмотрим операторы удвоенной проекции спина одной из частиц, образующих двухчастичное состояние, на направление, задаваемое вектором $\vec{a}=\left(a_{x}, a_{y}, a_{z}\right)$. Для первой частицы такой оператор имеет вид

$$
\hat{a} \otimes I=2\left(\vec{a}, \vec{S}^{(1)}\right) \otimes I,
$$

а для второй частицы

$$
I \otimes \hat{a}=2 I \otimes\left(\vec{a}, \vec{S}^{(2)}\right) .
$$

Здесь $I$ - единичный оператор в двумерном пространстве, натянутом на базис (28), $\hat{a}$ - оператор (29).

Величины (12) можно выразить через элементы матрицы плотности (27). Для этого в качестве векторов $\vec{a}, \vec{b}$, которые используются при измерении величины спиновой корреляции, будем выбирать всевозможные пары из тройки векторов $\vec{x}, \vec{y}, \vec{z}$, задающих координатные оси $(X, Y, Z)$. Легко видеть, что выполняются следующие соотношения:

$$
\begin{gathered}
\left(\begin{array}{ccc}
E(\vec{x}, \vec{x}) & E(\vec{x}, \vec{y}) & E(\vec{x}, \vec{z}) \\
E(\vec{y}, \vec{x}) & E(\vec{y}, \vec{y}) & E(\vec{y}, \vec{z}) \\
E(\vec{z}, \vec{x}) & E(\vec{z}, \vec{y}) & E(\vec{z}, \vec{z})
\end{array}\right)=\left(\begin{array}{ccc}
\operatorname{Sp}(\vec{x} \otimes \vec{x} \rho) & \operatorname{Sp}(\vec{x} \otimes \vec{y} \rho) & \operatorname{Sp}(\vec{x} \otimes \vec{z} \rho) \\
\operatorname{Sp}(\vec{y} \otimes \vec{x} \rho) & \operatorname{Sp}(\vec{y} \otimes \vec{y} \rho) & \operatorname{Sp}(\vec{y} \otimes \vec{z} \rho) \\
\operatorname{Sp}(\vec{z} \otimes \vec{x} \rho) & \operatorname{Sp}(\vec{z} \otimes \vec{y} \rho) & \operatorname{Sp}(\vec{z} \otimes \vec{z} \rho)
\end{array}\right)= \\
\quad=\left(\begin{array}{ccc}
\rho_{14}+\rho_{23}+\rho_{32}+\rho_{41} & i\left(\rho_{14}-\rho_{23}+\rho_{32}-\rho_{41}\right) & \rho_{13}+\rho_{31}-\rho_{24}-\rho_{42} \\
i\left(\rho_{14}+\rho_{23}-\rho_{32}-\rho_{41}\right) & -\rho_{14}+\rho_{23}+\rho_{32}-\rho_{41} & i\left(\rho_{13}-\rho_{31}-\rho_{24}+\rho_{42}\right) \\
\rho_{12}+\rho_{21}-\rho_{34}-\rho_{43} & i\left(\rho_{12}-\rho_{21}-\rho_{34}+\rho_{43}\right) & \rho_{11}-\rho_{22}-\rho_{33}+\rho_{44}
\end{array}\right) .
\end{gathered}
$$

Если последнюю матрицу обозначить буквой $P$, то выражение для среднего значения оператора спиновой корреляции примет вид

$$
E(\vec{a}, \vec{b})=\operatorname{Sp}(\hat{a} \otimes \hat{b} \rho)=\left(a_{x} a_{y} a_{z}\right) P\left(\begin{array}{l}
b_{x} \\
b_{y} \\
b_{z}
\end{array}\right)=(\vec{a}, P \vec{b}) .
$$

Матрица $P$ и соотношение (35) были найдены в работах [21].

Можно также измерять не корреляции проекций спинов двух частиц, а средние значения проекций спинов отдельных частиц, образующих пару. Средние значения операторов (32), (33), т.е. величи́ны (10), (11), имеют вид

$$
\begin{aligned}
& E(\vec{a})=2 S^{(1)}(\vec{a})=\operatorname{Sp}(\hat{a} \otimes I \rho)=(\vec{a}, \vec{L}), \\
& E(\vec{b})=2 S^{(2)}(\vec{b})=\operatorname{Sp}(I \otimes \hat{b} \rho)=(\vec{b}, \vec{R}) .
\end{aligned}
$$


Здесь $\vec{L}$ и $\vec{R}$ - векторы в трехмерном пространстве, задающиеся как

$$
\begin{gathered}
\vec{L}=\left(\begin{array}{l}
L_{x} \\
L_{y} \\
L_{z}
\end{array}\right)=\left(\begin{array}{c}
\operatorname{Sp}(\hat{x} \otimes I \rho) \\
\operatorname{Sp}(\hat{y} \otimes I \rho) \\
\operatorname{Sp}(\hat{z} \otimes I \rho)
\end{array}\right)=\left(\begin{array}{c}
\rho_{12}+\rho_{21}+\rho_{34}+\rho_{43} \\
i\left(-\rho_{12}+\rho_{21}-\rho_{34}+\rho_{43}\right) \\
\rho_{11}-\rho_{22}+\rho_{33}-\rho_{44}
\end{array}\right), \\
\vec{R}=\left(\begin{array}{l}
R_{x} \\
R_{y} \\
R_{z}
\end{array}\right)=\left(\begin{array}{c}
\operatorname{Sp}(I \otimes \hat{x} \rho) \\
\operatorname{Sp}(I \otimes \hat{y} \rho) \\
\operatorname{Sp}(I \otimes \hat{z} \rho)
\end{array}\right)=\left(\begin{array}{c}
\rho_{13}+\rho_{31}+\rho_{24}+\rho_{42} \\
i\left(-\rho_{13}+\rho_{31}-\rho_{24}+\rho_{42}\right) \\
\rho_{11}+\rho_{22}-\rho_{33}-\rho_{44}
\end{array}\right) .
\end{gathered}
$$

Таким образом, 15 независимых элементов матрицы плотности (27) можно разбить на три части: 9 элементов имеют смысл средних значений оператора спиновой корреляции для всевозможных пар координатных осей $X, Y, Z$, три элемента представляют собой средние значения проекции спина первой частицы на эти оси и еще три - средние значения проекции спина второй частицы на те же оси. Они образуют, соответственно, матрицу $P(34)$ и векторы $\vec{L}, \vec{R}(37)$.

По данным, содержащимся в компонентах матрицы $P(34)$ и векторов $\vec{L}, \vec{R}(37)$, можно восстановить все элементы матрицы плотности (27). Соответствующие формулы таковы:

$$
\begin{aligned}
\rho_{11} & =\frac{1}{4}\left(1+P_{33}+L_{3}+R_{3}\right), & \rho_{22} & =\frac{1}{4}\left(1-P_{33}-L_{3}+R_{3}\right), \\
\rho_{33} & =\frac{1}{4}\left(1-P_{33}+L_{3}-R_{3}\right), & \rho_{44} & =\frac{1}{4}\left(1+P_{33}-L_{3}-R_{3}\right), \\
\rho_{12} & =\frac{1}{4}\left(P_{31}+L_{1}+i\left(-P_{32}+L_{2}\right)\right), & \rho_{13} & =\frac{1}{4}\left(P_{13}+R_{1}+i\left(-P_{23}+R_{2}\right)\right), \\
\rho_{34} & =\frac{1}{4}\left(-P_{31}+L_{1}+i\left(P_{32}+L_{2}\right)\right), & \rho_{24} & =\frac{1}{4}\left(-P_{13}+R_{1}+i\left(P_{23}+R_{2}\right)\right), \\
\rho_{14} & =\frac{1}{4}\left(P_{11}-P_{22}-i\left(P_{12}+P_{21}\right)\right), & \rho_{23} & =\frac{1}{4}\left(P_{11}+P_{22}+i\left(P_{12}-P_{21}\right)\right) .
\end{aligned}
$$

В случае факторизуемых состояний формула (35) для величины спиновой корреляции может быть упрощена. Пусть

$$
\rho_{\mathrm{f}}=\rho^{\alpha} \otimes \rho^{\beta}=\left(\begin{array}{cc}
\rho_{11}^{\alpha} & \rho_{12}^{\alpha} \\
\rho_{21}^{\alpha} & \rho_{22}^{\alpha}
\end{array}\right) \otimes\left(\begin{array}{cc}
\rho_{11}^{\beta} & \rho_{12}^{\beta} \\
\rho_{21}^{\beta} & \rho_{22}^{\beta}
\end{array}\right),
$$

тогда матрицу (34) можно представить в виде произведения двух матриц:

$$
\begin{aligned}
& P=\left(P^{\alpha}\right)^{\mathrm{T}} P^{\beta}= \\
& =\left(\begin{array}{ccc}
\left(\rho_{12}^{\alpha}+\rho_{21}^{\alpha}\right) & 0 & 0 \\
i\left(\rho_{12}^{\alpha}-\rho_{21}^{\alpha}\right) & 0 & 0 \\
\left(\rho_{11}^{\alpha}-\rho_{22}^{\alpha}\right) & 0 & 0
\end{array}\right)\left(\begin{array}{ccc}
\left(\rho_{12}^{\beta}+\rho_{21}^{\beta}\right) & i\left(\rho_{12}^{\beta}-\rho_{21}^{\beta}\right) & \left(\rho_{11}^{\beta}-\rho_{22}^{\beta}\right) \\
0 & 0 & 0 \\
0 & 0 & 0
\end{array}\right) .
\end{aligned}
$$

Для таких матриц $\rho_{\mathrm{f}}$ формула (35) принимает вид

$$
\begin{aligned}
E(\vec{a}, \vec{b})= & \operatorname{Sp}(\hat{a} \otimes \hat{b} \rho)=\left(P^{\alpha} \vec{a}, P^{\beta} \vec{b}\right)= \\
=[ & \left.a_{x}\left(\rho_{12}^{\alpha}+\rho_{21}^{\alpha}\right)+i a_{y}\left(\rho_{12}^{\alpha}-\rho_{21}^{\alpha}\right)+a_{z}\left(\rho_{11}^{\alpha}-\rho_{22}^{\alpha}\right)\right] \times \\
& \quad \times\left[b_{x}\left(\rho_{12}^{\beta}+\rho_{21}^{\beta}\right)+i b_{y}\left(\rho_{12}^{\beta}-\rho_{21}^{\beta}\right)+b_{z}\left(\rho_{11}^{\beta}-\rho_{22}^{\beta}\right)\right]=E^{\alpha}(\vec{a}) E^{\beta}(\vec{b}) .
\end{aligned}
$$


Мы видим, что для факторизуемых состояний и матрица плотности (27), и компоненты матрицы $P(34)$ выражаются только через компоненты векторов $\vec{L}, \vec{R}(37)$. Используя полученные выражения для средних значений проекций спинов частиц и спиновой корреляции, можно также найти выражение для коэффициента корреляции (19) через элементы матрицы плотности (27) и координаты векторов $\vec{a}, \vec{b}$ :

$$
K_{\rho}(\vec{a}, \vec{b})=\frac{(\vec{a}, P \vec{b})-(\vec{L}, \vec{a})(\vec{R}, \vec{b})}{\sqrt{1-(\vec{L}, \vec{a})^{2}} \sqrt{1-(\vec{R}, \vec{b})^{2}}}
$$

С помощью выражения (35) неравенство (8) можно записать в следующем виде:

$$
\begin{aligned}
|E(\vec{a}, \vec{b})-E(\vec{a}, \vec{c})| & =|(\vec{a}, P \vec{b})-(\vec{a}, P \vec{c})|= \\
& =|(\vec{a}, P(\vec{b}-\vec{c}))| \leqslant|P(\vec{b}-\vec{c})| \leqslant \sqrt{2} \sup _{\vec{n}}|P \vec{n}|, \quad|\vec{n}|=1 .
\end{aligned}
$$

Используя соотношения (35), (36), можно выразить правую часть неравенства (26) через элементы матрицы плотности (27) и свести это неравенство к обобщенному неравенству БКХШХ [21], [22]:

$$
|E(\vec{a}, \vec{b})+E(\vec{a}, \vec{c})+E(\vec{d}, \vec{b})-E(\vec{d}, \vec{c})| \leqslant \sqrt{2} \sup _{\vec{n}_{1}, \vec{n}_{2}}\left(\left|P \vec{n}_{1}\right|+\left|P \vec{n}_{2}\right|\right)
$$

здесь точная верхняя грань берется по всем парам векторов $\vec{n}_{1}, \vec{n}_{2}$ таким, что

$$
\left(\vec{n}_{1}, \vec{n}_{2}\right)=0, \quad\left|\vec{n}_{1}\right|=\left|\vec{n}_{2}\right|=1 .
$$

В случае ЭПР-состояния $\Psi_{0,0}=\frac{1}{\sqrt{2}}(|+\rangle|-\rangle-|-\rangle|+\rangle)$

$$
\begin{gathered}
\rho_{0,0}=\frac{1}{2}\left(\begin{array}{cccc}
0 & 0 & 0 & 0 \\
0 & 1 & -1 & 0 \\
0 & -1 & 1 & 0 \\
0 & 0 & 0 & 0
\end{array}\right), \quad P_{0,0}=\left(\begin{array}{ccc}
-1 & 0 & 0 \\
0 & -1 & 0 \\
0 & 0 & -1
\end{array}\right), \quad L_{0,0}=R_{0,0}=\left(\begin{array}{c}
0 \\
0 \\
0
\end{array}\right) ; \\
E(\vec{a})=E(\vec{b})=0, \quad K_{0,0}(\vec{a}, \vec{b})=E(\vec{a}, \vec{b})=-(\vec{a}, \vec{b}) .
\end{gathered}
$$

Если $\vec{a}=\vec{b}$, то $K_{0,0}(\vec{a}, \vec{a})=-1$, поэтому в данном случае наборы чисел

$$
\left\{v_{i}(\vec{a}) ; i=1, \ldots, N\right\}, \quad\left\{w_{i}(\vec{b}) ; i=1, \ldots, N\right\}
$$

не являются независимыми друг от друга. Из соотношений (46) следует, что в каждом из наборов (47) половина членов равна 1 , а вторая половина равна -1 . При этом внутри одного набора, например внутри $\left\{v_{i}(\vec{a}) ; i=1, \ldots, N\right\},+1$ и -1 могут быть расположены произвольным образом, но это расположение однозначно задает расположение чисел +1 и -1 внутри набора $\left\{w_{i}(\vec{a}) ; i=1, \ldots, N\right\}$. Это соответствие таково, что если $v_{i}(\vec{a})=1$, то $w_{i}(\vec{a})=-1$, а если $v_{i}(\vec{a})=-1$, то $w_{i}(\vec{a})=1$.

Но если мы имеем два набора $\left\{v_{i}^{1}(\vec{a}) ; i=1, \ldots, N\right\}$ и $\left\{w_{i}^{2}(\vec{a}) ; i=1, \ldots, N\right\}$, полученные в разных сериях измерений, то для них такого соответствия не существует, и значение величины $v_{i}^{1}$ никак не коррелирует со значением величины $w_{i}^{2}$. Поэтому если с их помощью построить сумму (12), то это не будет спиновая корреляция $E(\vec{a}, \vec{a})$. 


\section{6. ЗАКЛЮЧЕНИЕ}

Проведенный анализ показывает, что факт нарушения в экспериментах неравенства Белла (8) и неравенства БКХШХ (22) связан с тем, что эти неравенства доказаны для корреляционных средних, которые вычислены для произвольных случайных величин, а при проверке используются корреляционные средние, в которые входят случайные величины, относящиеся к одной коррелированной паре одночастичных состояний. Для таких корреляционных средних выполняются не неравенства (8), (22), а неравенства (20), (26), которые включают в себя коэффициенты корреляции соответствующих случайных величин. Мы называем их корреляционными неравенствами Белла. Если коэффициенты корреляции равны нулю, то корреляционные неравенства (20), (26) переходят в обычные неравенства Белла (8), (22). Это имеет место в том случае, когда двухчастичное состояние является факторизуемым или сепарабельным. Формула (42) дает явное выражение для коэффициента корреляции (19) через элементы матрицы плотности (27) двухчастичного состояния и координаты векторов $\vec{a}, \vec{b}$, определяющих направления, на которые проецируются спины частиц.

Наш подход близок к тому, который был развит в книге [7]. Детально все вопросы сходства и различия подходов будут исследованы в отдельной работе. Также специального разбора, основанного на результатах данной работы, требуют проблемы нелокальности квантовой механики и скрытых переменных.

Благодарности. Автор благодарен И. В. Воловичу, В. И. Манько и А. Ю. Хренникову за многочисленные дискуссии, способствовавшие углублению понимания данной проблемы, а также М. Купчинскому, обратившему его внимание на работы [19], и О.А. Ивановой за консультации по теории вероятностей.

\section{Список литературы}

[1] А. Эйнштейн, Б. Подольский, Н. Розен, УФН, 16:4 (1936), 440-446.

[2] Н. Бор, УФH, 16:4 (1936), 446-457.

[3] J. S. Bell, Physics, 1:3 (1964), 195-200.

[4] J. S. Bell, Rev. Modern Phys., 38:3 (1966), 447-452.

[5] J. F. Clauser, M. A. Horne, A. Shimony, R. A. Holt, Phys. Rev. Lett., 23:15 (1969), 880-884.

[6] I. Volovich, Towards quantum information theory in space and time, arXiv: quant-ph/0203030; A. Khrennikov, I. Volovich, Local realism, contextualism and loopholes in Bell's experiments, arXiv: quant-ph/0212127; A. Khrennikov, I. V. Volovich, "Quantum nonlocality, EPR model and Bell's theorem", Proc. III Int. Sakharov Conf. on Physics, V. II, eds. A. Semikhatov, M. Vasiliev, V. Zaikin, World Sci., Singapore, 2003, 260-267.

[7] А. Ю. Хренников, Неколмогоровские теории вероятностей и квантовая физика, Физматлит, М., 2003.

[8] А. Боум, Квантовая механика. Основы и приложения, Мир, М., 1990.

[9] М. Нильсен, И. Чанг, Квантовые вычисления и квантовая информачия, Мир, М., 2006.

[10] M. Genovese, Phys. Rep., 413:6 (2005), 319-396.

[11] A. Khrennikov, Bell's inequality: physics meets probability, arXiv: 0709.3909.

[12] F. J. Belinfante, A Survey of Hidden-Variables Theories, Internat. Ser. Monogr. in Natural Philosophy, 55, Pergamon Press, Oxford, 1973.

[13] S. J. Freedman, J. F. Clauser, Phys. Rev. Lett., 28:14 (1972), 938-941. 
[14] A. Aspect, P. Grangier, G. Roger, Phys. Rev. Lett., 47:7 (1981), 460-463.

[15] A. Aspect, P. Grangier, G. Roger, Phys. Rev. Lett., 49:2 (1982), 91-94.

[16] A. Aspect, J. Dalibard, G. Roger, Phys. Rev. Lett., 49:25 (1982), 1804-1807.

[17] Z. Y. Ou, L. Mandel, Phys. Rev. Lett., 61:1 (1988), 50-53.

[18] T.E. Kiess, Y.H. Shih, A.V. Sergienko, C. O. Alley, Phys. Rev. Lett., 71:24 (1993), 3893-3897.

[19] M. Kupczynski, Phys. Lett. A, 121:2 (1987), 51-53; J. Russ. Laser Res., 26:6 (2005), $514-523$.

[20] А. В. Белинский, Д. Н. Клышко, УФН, 163:8 (1993), 1-45.

[21] В. А. Андреев, В.И. Манько, Писъма в ЖЖЭТФ, 72:2 (2000), 130-135; V. A. Andreev, V. I. Man'ko, Phys. Lett. A, 281:5-6 (2001), 278-288.

[22] В. А. Андреев, ТМФ, 152:3 (2007), 488-501.

Поступила в редакцию 13.02.2008, после доработки 21.05.2008 